\title{
Thermodynamic behaviour of undercooled melts
}

\author{
R K MISHRA and K S DUBEY \\ Department of Applied Physics, Institute of Technology, Banaras Hindu University, Varanasi \\ 221005 , India
}

MS received 24 November 1995; revised 19 December 1995

\begin{abstract}
The Gibbs free energy difference $(\Delta G)$ between the undercooled liquid and the equilibrium solid phases has been studied for the various kinds of glass forming melts such as metallic, molecular and oxides melts using the hole theory of liquids and an excellent agreement is found between calculated and experimental values of $\Delta G$. The study is made for non-glass forming melts also. The temperature dependence of enthalpy difference $(\Delta H)$ and entropy difference $(\Delta S)$ between the two phases, liquid and solid, has also been studied. The Kauzmann temperature $\left(T_{0}\right)$ has been estimated using the expression for $\Delta S$ and a linear relation is found between the reduced glass transition temperature $\left(T_{g} / T_{\mathrm{m}}\right)$ and $\left(T_{0} / T_{\mathrm{m}}\right)$. The residual entropy $\left(\Delta S_{\mathrm{R}}\right)$ has been estimated for glass forming melts and an attempt is made to correlate $\Delta S_{\mathrm{R}}, T_{\mathrm{g}}, T_{0}$, and $T_{\mathrm{m}}$ which play a very important role in the study of glass forming melts.
\end{abstract}

Keywords. Undercooled melts; Gibbs free energy; Kauzmann temperature; metallic glasses; residual entropy.

\section{Introduction}

The production of metallic glasses by rapid solidification techniques has recently received sufficient attention and resulted in a revival of interests in nucleation and growth kinetics in liquid alloys, The Gibbs free energy difference $(\Delta G)$ between the undercooled liquid and the equilibrium solid phases is found to be an important parameter in the classical theory of nucleation and growth processes while the entropy difference $(\Delta S)$ between the liquid and solid phases plays an important role in the viscosity of these melts. The Gibbs free energy difference $(\Delta G)$ can be estimated by using the thermodynamic relations from a knowledge of the experimentally measured values of specific heat difference $(\Delta C p)$ between the undercooled melt and equilibrium solid phases. But the experimental measurements of the specific heat of the glass forming melts in their super-cooled states can be carried out only in a restricted temperature range below the melting temperature $\left(T_{\mathrm{m}}\right)$ due to their strong tendency to crystallization which prevents any direct measurements of the thermodynamic quantities. It can at best be measured near the melting temperature and at the glass transition temperature $\left(T_{\mathrm{g}}\right)$. Often, the behaviour of the thermodynamic parameters from $T_{\mathrm{g}}$ to $T_{\mathrm{m}}$ is obtained by extrapolating the high temperature data to low temperature region.

Due to experimental problems, several investigators (Turnbull 1950; Hoffman 1958; Jones and Chadwick 1971; Thompson and Spaepen 1979) have tried to suggest appropriate analytical expressions for $\Delta G$ in terms of experimentally measured parameters such as entropy of fusion $\left(\Delta S_{\mathrm{m}}\right), T_{\mathrm{m}}$, etc. Most of these expressions are inadequate for describing the temperature dependence of $\Delta G$ over a large degree of undercoolings.

Recently an expression for $\Delta G$ has been obtained by Dubey and Ramachandrarao (1984) based on the hole theory of liquids. Following the earlier work of Dubey and his coworkers (Dubey and Ramachandrarao 1984: Pandey et al 1987), the aim of the 
present work is to study $\Delta G$ for various kinds of glass forming melts such as metallic, molecular and oxide glass formers. $\Delta G$ has been estimated for $A u_{77} G_{18.6} S_{9.4}$, $\mathrm{Au}_{81.4} \mathrm{Si}_{18.6}, \mathrm{Mg}_{85.5} \mathrm{Cu}_{14.5}, \mathrm{Mg}_{81.6} \mathrm{Ga}_{18.4}$, glycerol, ethanol, 2-methyl pentane, $\mathrm{B}_{2} \mathrm{O}_{3}, \mathrm{SiO}_{2}$ and $\mathrm{GeO}_{2}$ in the temperature range $T_{\mathrm{g}}$ to $T_{\mathrm{m}}$ and an excellent agreement is obtained between calculated and experimental values of $\Delta G$. The study is also made for three samples of non-glass forming melts, $\mathrm{Pb}, \mathrm{Ga}$ and $\mathrm{Bi}_{55} \mathrm{~Pb}_{45}$, in the temperature range $0.5 T_{\mathrm{m}}$ to $T_{\mathrm{m}}$. The expressions for the entropy difference $(\Delta S)$ and enthalpy difference $(\Delta H)$ between the liquid and solid phases have been derived using the hole theory of liquids and the applicability of the expression obtained has been tested by estimating $\Delta S$ and $\Delta H$ for one sample from each kind of glass forming melts $\mathrm{Mg}_{85.5} \mathrm{Cu}_{14.5}$ (metallic), glycerol (molecular), and $\mathrm{SiO}_{2}$ (oxide) and one sample of non-glass forming melt, $\mathrm{Pb}$. A very good agreement is found between calculated and experimental data.

The expression derived for $\Delta S$ has been further used to obtain an expression for the Kauzmann temperature $T_{0}$ (which is also known as the ideal glass transition temperature) as well as for the residual entropy $\left(\Delta S_{R}\right)$ of glass forming melts and $T_{0}$ and $\Delta S_{R}$ are calculated for the above stated glass forming melts. Attempt has been made to correlate $T_{0}$ and $T_{\mathrm{g}}$ and a linear relation has been obtained between $T_{\mathrm{g}} / T_{\mathrm{m}}$ and $T_{0} / T_{\mathrm{m}}$ for each kind of glass forming melts. The role of the characteristic temperatures $T_{0}, T_{\mathrm{g}}$ and $T_{\mathrm{m}}$ in the estimation of the 'frozen-in' entropy $\left(\Delta S_{R}\right)$ has been studied and a linear relation is found between $\Delta S_{\mathrm{R}} / \Delta S_{\mathrm{m}}$ and $\left(T_{\mathrm{g}}-T_{0}\right) / T_{\mathrm{m}}$. Attempt has also been made to study the variation of the residual entropy with the ratio $\left(\Delta C p^{m} / \Delta S_{\mathrm{m}}\right)$ of the specific heat and entropy difference between two liquid and solid phases, at $T=T_{\mathrm{m}}$. The glass forming ability of material has been discussed in terms of the residual entropy of melts.

\section{Free energy, enthalpy and entropy of undercooled liquids}

According to the hole theory, a liquid state is described as a quasi-crystalline lattice with a considerable fraction of vacant sites of holes and the hole concentration is usually derived by minimizing the change in the free energy associated with the introduction of holes into a lattice. Following Frenkel (1955) as well as Hirai and Eyring $(1958,1959)$, the change in the free energy $(\Delta G)$ due to introduction of $N_{\mathrm{h}}$ hole into a lattice having $N_{\alpha}$ atoms or molecules at a temperature $T$ can be expressed as

$$
\Delta G=N_{\mathrm{h}}\left(\varepsilon_{\mathrm{h}}+p v_{\mathrm{h}}\right)+K_{\mathrm{B}} T\left[N_{\mathrm{h}} \ln \frac{N_{\mathrm{h}}}{N_{\mathrm{h}}+n N_{\alpha}}+N_{\mathrm{h}} \ln \frac{n N_{\alpha}}{N_{\mathrm{h}}+n N_{\alpha}}\right]-T \Delta S_{\mathrm{h}},
$$

here $\varepsilon_{\mathrm{h}}$ is the energy required for the formation of a hole of volume $v_{\mathrm{h}}, p$ the external pressure, $K_{\mathrm{B}}$ represents the Boltzmann constant, $n=v_{\alpha} / v_{\mathrm{h}}$ is a measure of the relative volume of a hole and an atom, $v_{\alpha}$ the hard core volume per atom or molecule and $\Delta S_{h}$ the entropy change associated with the formation of a hole.

Following Flory (1953), Sanchez (1974) and Dubey and Ramachandrarao (1984) and minimizing the $\Delta G$, an expression for $N_{\mathrm{h}}$ can be obtained as

$$
\begin{aligned}
& N_{\mathrm{h}}=n N_{\alpha}(g /(1-g)), \\
& g=\exp \left\{-\left(\frac{\varepsilon_{\mathrm{h}}+p v_{\mathrm{h}}}{K_{\mathrm{B}} T}\right)-A\right\},
\end{aligned}
$$


with

$$
A=1-1 / n
$$

The temperature dependence of $\Delta C p$ can be derived by realizing $\Delta C p=\mathrm{d} / \mathrm{d} t(\Delta H)$. According to Flory (1953) and Sanchez (1974), the enthalpy change associated with the formation of hole is given by

$$
\Delta H=(1-g) \varepsilon_{\mathrm{h}} N_{\mathrm{h}},
$$

and can be used to obtain the temperature dependence of the specific heat difference between the liquid and solid phases. Realising $\Delta C p=\mathrm{d} / \mathrm{d} t(\Delta H)$, and following Dubey and Ramachandrarao (1984) one can have

$$
\Delta C p=n R\left(\frac{E_{\mathrm{h}}}{R T}\right)^{2} g
$$

here $R$ is the gas constant and $E_{\mathrm{h}}$ the hole formation energy per mole.

It is usually found that $\Delta C p$ increases with increase in the undercooling below $T_{\mathrm{m}}$. The process of increase in $\Delta C p$ cannot go on indefinitely and as per Kauzmann paradox, $\Delta C p$ should attain a maximum value at some undercooled temperature to avoid the entropy catastrophe. The temperature at which $\Delta C p$ becomes maximum can be assumed to be the same at which $\Delta S$ becomes zero, i.e. the ideal glass transition temperature $T_{0}$. As a result. one gets

$$
E_{\mathrm{h}} / R T_{0}=2
$$

Similar relation has also been obtained by Sanchez (1974) in connection with the viscous behaviour of polymeric melts. Consequently, (4) reduces to

$$
\Delta C p=\Delta C p^{m}\left(T_{\mathrm{m}} / T\right)^{2} e^{-2 \delta \Delta T / T},
$$

with $\delta=T_{0} / T_{\mathrm{m}}$ is the reduced ideal glass transition temperature.

The expression for $\Delta C p$ stated in (6) can be used to derive an expression for $\Delta G$ with the aid of basic thermodynamic relations

$$
\begin{aligned}
& \Delta G=\Delta H-T \Delta S, \\
& \Delta H=\Delta H_{\mathrm{m}}-\int_{T}^{T_{\mathrm{m}}} \Delta C p \mathrm{~d} T . \\
& \Delta S=\Delta S_{\mathrm{m}}-\int_{T}^{T_{\mathrm{m}}}(\Delta C p / T) \mathrm{d} T .
\end{aligned}
$$

Appropriate substitution of $(6)$ and simplification of (7) yield an expression for $\Delta G$ as

$$
\Delta G=\Delta S_{\mathrm{m}} \Delta T-\frac{\Delta C p^{m}}{4 \delta^{2}}\left[2 \delta \Delta T-T\left(1-e^{2 \delta \Delta T / T}\right)\right]
$$

under the approximations,

$$
1-e^{2 x}=2 x \frac{3-x}{3+2 x}
$$


and

$$
e^{2 x}-1=2 x \frac{3+x}{3-2 x}
$$

the expression for $\Delta G$ stated in (10) reduces to

$$
\Delta G=\Delta S_{\mathrm{m}} \Delta T-\Delta C p^{m} \frac{\Delta T^{2}}{2 T\left(1+\frac{2}{3}(\delta \Delta T / T)\right)},
$$

which can further be simplified as

$$
\Delta G=\Delta S_{\mathrm{m}} \Delta T-\Delta C p^{m} \frac{\Delta T^{2}}{2 T}\left(1-\frac{2}{3} \delta \frac{\Delta T}{T}\right) .
$$

The expression stated in (13) requires the knowledge of $\Delta C p^{m}, \Delta S_{\mathrm{m}}$ and $T_{\mathrm{m}}$ which can be measured easily. The parameter $\delta$ can also be estimated with the aid of $\Delta C p^{m}$ and $\Delta S_{\mathrm{m}}$ which has been discussed in the next section.

The use of (6) and (9) yields an expression for $\Delta S$ as

$$
\Delta S=\Delta S_{\mathrm{m}}-\frac{\Delta C p^{m}}{4 \delta^{2}}\left[(1+2 \delta)-\left(1+2 \delta\left(T_{\mathrm{m}} / T\right)\right) e^{-2 \delta \Delta T / T}\right] .
$$

In view of the approximation stated in (11), expression for $\Delta S$ takes the form

$$
\Delta S=\Delta S_{\mathrm{m}}-\frac{\Delta C p^{m} \Delta T\left(T_{\mathrm{m}}+T\right)}{2 T^{2}}\left[\frac{1-(2 / 3) \delta(\Delta T / T)\left(T_{\mathrm{m}} /\left(T_{\mathrm{m}}+T\right)\right)}{1+(2 / 3) \delta(\Delta T / T)}\right],
$$

which can be further approximated as

$$
\Delta S=\Delta S_{\mathrm{m}}-\frac{\Delta C p^{m} \Delta T\left(T_{\mathrm{m}}+T\right)}{2 T^{2}}\left[1-\frac{2}{3} \delta \frac{\Delta T}{T}\left(\frac{2 T_{\mathrm{m}}+T}{T_{\mathrm{m}}+T}\right)\right] .
$$

The expression for $\Delta H$ can also be derived with the aid of (6) and (8) and takes the form

$$
\Delta H=\Delta H_{\mathrm{m}}-\frac{\Delta C p^{m} T_{\mathrm{m}}}{2 \delta}\left(1-e^{-2 \delta \Delta T / T}\right)
$$

Use of approximation stated in (11) yields to a more simplified form

$$
\Delta H=\Delta H_{\mathrm{m}}-\Delta C p^{m} \frac{\Delta T}{T} T_{\mathrm{m}}\left(1-\delta \frac{\Delta T}{T}\right) .
$$

Thus, $\Delta S$ and $\Delta H$ can be estimated with the aid of $\Delta S_{\mathrm{m}}, \Delta C p^{m}$ and $T_{\mathrm{m}}$ which can be measured easily.

\section{The ideal glass transition temperature $T_{0}$ and the residual entropy $\Delta S_{\mathrm{R}}$}

The problem associated with a purely kinetic definition of the glass transition was pointed out by Kauzmann (1948). One of the problems is $\Delta S$ becoming negative at some 
temperature below $T_{\mathrm{g}}$. He further argued that a liquid loses its entropy at a faster rate than the corresponding equilibrium solid. Consequently one can assume an isoentropic temperature $\left(T_{0}\right)$ at which both liquid and solid phases have equal entropy. Gibbs and DiMarzio (1958) as well as Adam and Gibbs (1965) have pleaded that such a transition should be of the second order while Cohen and Grest (1979) reported in favour of the first order. Recalling (14) and setting to zero at $T=T_{0}$, one can have an expression for the estimation of $T_{0}$ in terms of $\Delta C p^{m}, \Delta S_{\mathrm{m}}$ and $T_{\mathrm{m}}$ of the form

$$
\Delta S_{\mathrm{m}}-\frac{\Delta C p^{m}}{2 \delta}\left[(1+2 \delta)-3 e^{-2(1-\delta)}\right]=0
$$

The solution of the transcendental equation can be achieved by iterative procedure. However an approximate and more simplified form of the expression for estimating $\delta$ can be obtained under the approximation stated in (11) as

$$
4 \delta^{3}-\left[10+\frac{5 \Delta C p^{m}}{\Delta S_{\mathrm{m}}}\right] \delta^{2}+\frac{4 \Delta C p^{m}}{\Delta S_{\mathrm{m}}} \delta+\frac{\Delta C p^{m}}{\Delta S_{\mathrm{m}}}=0,
$$

which shows that the value of $\delta$ is entirely governed by the ratio $\Delta C p^{m} / \Delta S_{\mathrm{m}}$. It may be noted that generally $\delta^{3}$ is found to be much smaller compared to $\delta^{2}$ and an approximate value of the ideal glass transition temperature $T_{0}$ is given by

$$
T_{0}=T_{\mathrm{m}} \frac{2 s+\left(9 s^{2}+10 s\right)^{1 / 2}}{5(2+s)}
$$

where

$$
s=\frac{\Delta C p^{m}}{\Delta S_{\mathrm{m}}}
$$

According to the statistico-mechanical quasi-lattice theory of Gibbs and DiMarzio (1958), the configurational entropy becomes zero at the iso-entropic temperature $T_{0}$. The apparent entropy difference between the hypothetical glass and crystal at $T-0 \mathrm{~K}$ is the same as the configurational entropy of the liquid frozen-in at $T_{\mathrm{g}}$. In other words, it can also be understood that some amount of entropy is blocked in the glassy configuration at $T_{\mathrm{g}}$ and is usually referred to as the residual entropy or 'frozen-in' entropy. Thus the residual entropy is given by

$$
\begin{aligned}
\Delta S_{\mathrm{R}} & =\Delta S \quad(\text { at } T=0 \mathrm{~K})=\Delta S\left(T_{\mathrm{g}}\right) \\
& =\frac{\Delta C p^{m}}{4 \delta^{2}}\left[\left(1+2 \delta \frac{T_{\mathrm{m}}}{T_{\mathrm{g}}}\right) e^{-2 \delta \Delta T_{\mathrm{g}} / T_{\mathrm{g}}}-3 e^{-2(1-\delta)}\right],
\end{aligned}
$$

where

$$
\Delta T_{\mathrm{g}}=\left(T_{\mathrm{m}}-T_{\mathrm{g}}\right)
$$

Use of (11) yields a simplified form of $\Delta S_{R}$ as

$$
\Delta S_{\mathbf{R}}=\Delta C p^{m}\left[1 \cdot 5-2\left(T_{0} / T_{\mathrm{g}}\right)+0 \cdot 5\left(T_{0} / T_{\mathrm{g}}\right)^{2}\right] e^{-2(1-\delta)},
$$

which can also be stated as

$$
\Delta S_{\mathrm{R}}=\Delta S_{\mathrm{m}}\left[1-\frac{\Delta C p^{m}}{\Delta S_{\mathrm{m}}}\left\{T_{\mathrm{gr}}^{-2}-1-\frac{2}{3} \delta\left(2 T_{\mathrm{gr}}^{-3}-3 T_{\mathrm{gr}}^{-2}-1\right)\right\}\right],
$$


as a function of $\Delta C p^{m}$ and $\Delta S_{\mathrm{m}}$. Here $T_{\mathrm{gr}}=T_{\mathrm{g}} / T_{\mathrm{m}}$ is the reduced glass transition temperature.

Expressions stated in $(21-24)$ show that the residual entropy is mainly governed by the characteristic temperatures $T_{\mathrm{g}}, T_{\mathrm{m}}$ and $T_{0}$.

\section{Results and discussions}

The experimental values of $\Delta G$ have been calculated with the help of experimentally measured values of $\Delta C p$ and using (7). Generally, experimental data of $\Delta C p$ is represented by the equation

$$
\Delta C p=A+B T+C T^{2}+D / T+E / T^{2},
$$

where $A, B, C, D$ and $E$ are constants. The material constants are reported in tables 1 a and $b$. To test the expression for $\Delta G$ reported in (13), $\Delta G$ has been calculated for various types of glass forming melts in the temperature range $T_{\mathrm{m}}$ to $T_{\mathrm{g}}$, which includes $\mathrm{Au}_{77} \mathrm{Ge}_{13.6} \mathrm{Si}_{9.4}, \mathrm{Au}_{81.4} \mathrm{Si}_{18.6}, \mathrm{Mg}_{85.5} \mathrm{Cu}_{14.5}$ and $\mathrm{Mg}_{81.6} \mathrm{Ga}_{18.4}$ (metallic glass formers), glycerol, 2-methyl pentane and ethanol (molecular glass formers) and $\mathrm{B}_{2} \mathrm{O}_{3}$, $\mathrm{GeO}_{2}$ and $\mathrm{SiO}_{2}$ (oxide glass formers) and results obtained are illustrated in figures 1-3 for metallic, molecular and oxide glass forming melts respectively. To see the applicability of the expression reported in (13) for non-glass forming melts, $\Delta G$ is also calculated for $\mathrm{Ga}, \mathrm{Pb}$ and $\mathrm{Bi}_{55} \mathrm{~Pb}_{45}$ in the temperature range $T_{\mathrm{m}}$ to $T_{\mathrm{m}} / 2$ and results obtained are shown in figure 4 . An excellent agreement between calculated and experimental values of $\Delta G$ can be seen in figures $1-4$ for the glass forming as well as for non-glass forming melts.

The temperature dependence of the thermodynamic parameters $\Delta S$ and $\Delta H$ has been studied for one sample of each group of glass forming melts; $\mathrm{MgCu}$ (metallic), glyceral (molecular), $\mathrm{SiO}_{2}$ (oxide) in the temperature range $T_{\mathrm{m}}$ to $T_{\mathrm{g}}$ and one sample of non-glass forming melt $\mathrm{Pb}$ in the temperature range $T_{\mathrm{m}}$ to $T_{\mathrm{m}} / 2$ and are illustrated in figures $5-8$ respectively. From figures $5-8$, it can be seen that the agreement between calculated and experimental values of $\Delta H$ is very good for glass forming as well as for non-glass forming melts. With the help of these figures (figures 5-8), it can also be realized that the calculated values of $\Delta S$ are quite close to the experimental values for materials under study except $\mathrm{Pb}$. The agreement between the calculated and experimental values of $\Delta S$ is not very satisfactory for $\mathrm{Pb}$ at the large undercoolings. As a result of it, one can say that expressions reported in (16) and (18) are capable to explain the temperature dependence of the thermodynamic parameters $\Delta S$ and $\Delta H$ respectively. To see the goodness of the expression reported in (13) for $\Delta G$, a comparative study is made in table 2 for $\mathrm{Mg}_{85.5} \mathrm{Cu}_{14.5}$ with the results obtained using the expressions obtained by earlier workers (Turnbull 1950; Hoffman 1958; Thompson and Spaepen 1979) as

$$
\begin{aligned}
& \Delta G=\Delta S_{\mathrm{m}} \Delta T \\
& \Delta G=\Delta S_{\mathrm{m}} \Delta T \frac{T}{T_{\mathrm{m}}}, \\
& \Delta G=\Delta S_{\mathrm{m}} \Delta T \frac{2 T}{\left(T_{\mathrm{m}}+T\right)}
\end{aligned}
$$




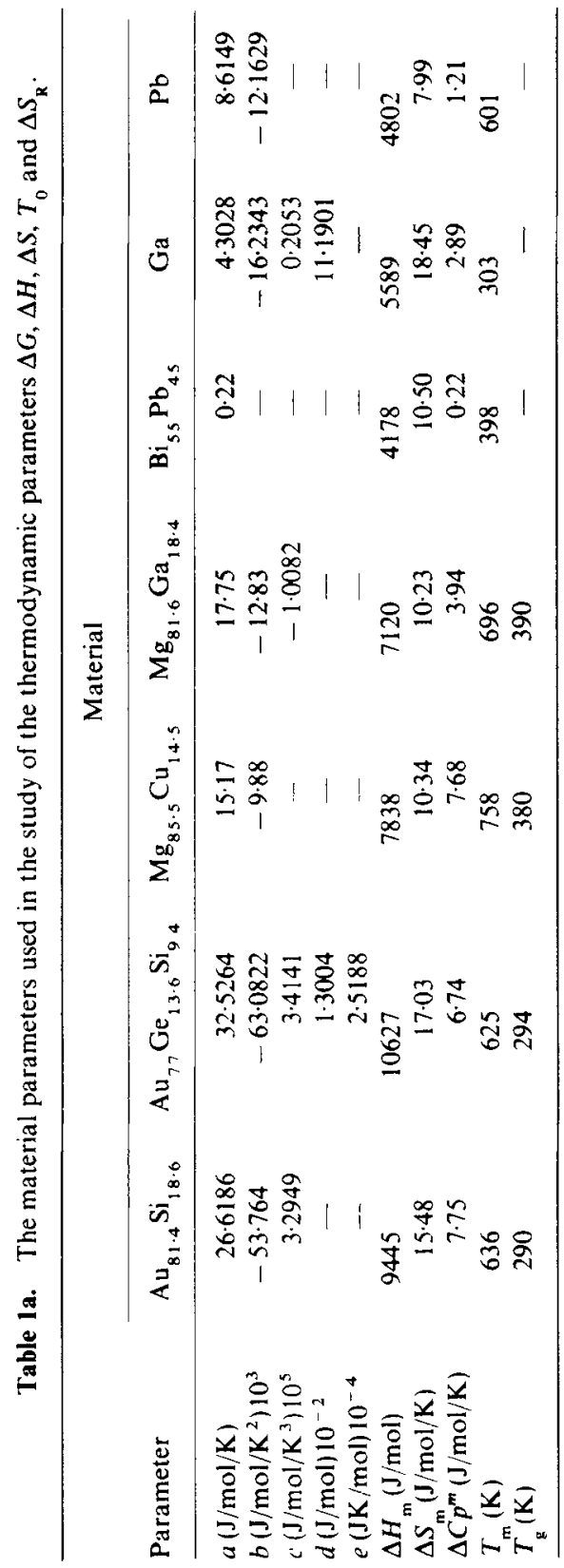


Table 1 b. The material parameters used in the study of the thermodynamic parameters $\Delta G$, $\Delta H, \Delta S, T_{0}$ and $\Delta S_{\mathbf{R}}$.

\begin{tabular}{lcccccc}
\hline & \multicolumn{7}{c}{ Material } \\
\cline { 2 - 7 } Parameter & Glycerol & Ethanol & $\begin{array}{l}\text { 2-methyl } \\
\text { pentane }\end{array}$ & $\mathrm{B}_{2} \mathrm{O}_{3}$ & $\mathrm{SiO}_{2}$ & $\mathrm{GeO}_{2}$ \\
\hline$a(\mathrm{~J} / \mathrm{mol} / \mathrm{K})$ & $90 \cdot 8646$ & $51 \cdot 5810$ & $146 \cdot 871$ & $70 \cdot 854$ & $14 \cdot 20$ & $20 \cdot 98$ \\
$b\left(\mathrm{~J} / \mathrm{mol} / \mathrm{K}^{2}\right) 10^{3}$ & $39 \cdot 06$ & $-167 \cdot 3$ & $-798 \cdot 5$ & $-73 \cdot 416$ & $-1 \cdot 9$ & $-9 \cdot 87$ \\
$c\left(\mathrm{~J} / \mathrm{mol} / \mathrm{K}^{3}\right) 10^{5}$ & - & - & - & - & - & - \\
$d(\mathrm{~J} / \mathrm{mol}) 10^{-2}$ & - & - & - & - & - & - \\
$e(\mathrm{JK} / \mathrm{mol}) 10^{-4}$ & - & - & - & $141 \cdot 12$ & $392 \cdot 1$ & $177 \cdot 7$ \\
$\Delta H_{\mathrm{m}}(\mathrm{J} / \mathrm{mol})$ & 18371 & 5015 & 6260 & 22261 & 9621 & 44517 \\
$\Delta S_{\mathrm{m}}(\mathrm{J} / \mathrm{mol} / \mathrm{K})$ & $62 \cdot 70$ & $31 \cdot 64$ & $52 \cdot 36$ & $30 \cdot 79$ & $4 \cdot 82$ & $32 \cdot 05$ \\
$\Delta C p^{m}(\mathrm{~J} / \mathrm{mol} / \mathrm{K})$ & $79 \cdot 42$ & $25 \cdot 06$ & $51 \cdot 41$ & $20 \cdot 47$ & $11 \cdot 39$ & $8 \cdot 19$ \\
$T_{\mathrm{m}}(\mathrm{K})$ & 293 & $158 \cdot 5$ & 119.55 & 723 & 1996 & 1389 \\
$T_{\mathbf{g}}(\mathrm{K})$ & 186 & 91 & 79.5 & 550 & 1443 & 823 \\
\hline
\end{tabular}

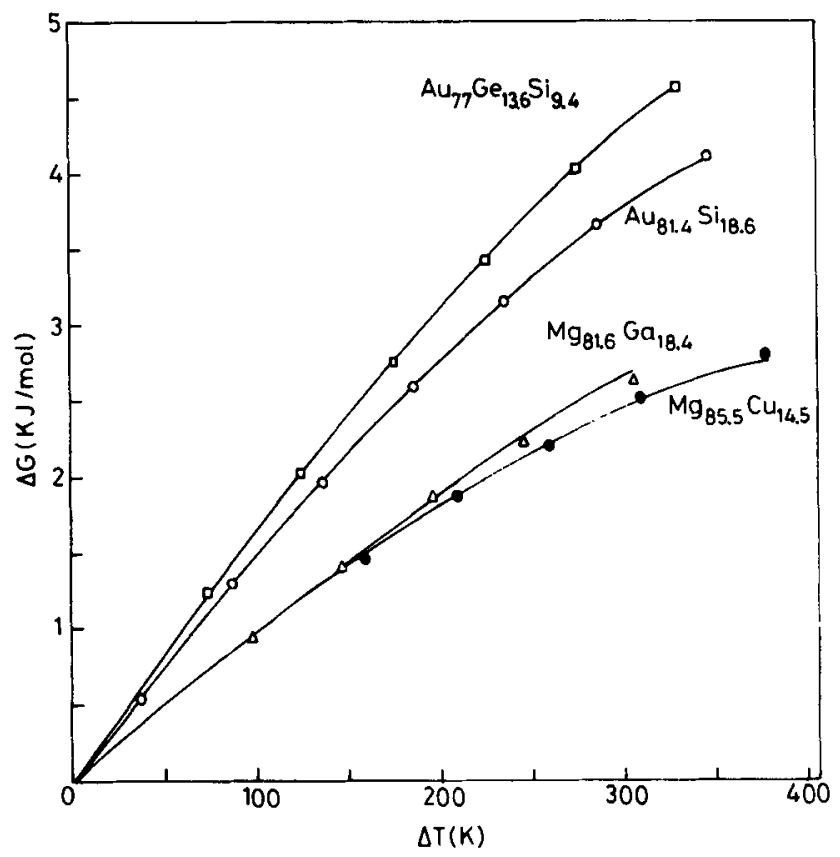

Figure 1. The Gibbs free energy difference, $\Delta G$, for metallic glass formers. Closed circles, triangles, open circles and squares represent experimental values of $\Delta G$ for $\mathrm{Mg}_{85.5} \mathrm{Cu}_{14.5}$, $\mathrm{Mg}_{81.6} \mathrm{Ga}_{18 \cdot 4}, \mathrm{Au}_{81 \cdot 4} \mathrm{Si}_{18.6}, \mathrm{Au}_{77} \mathrm{Ge}_{13.6} \mathrm{Si}_{9.4}$ respectively whereas lines are calculated values of the respective samples.

From table 2, it can be seen that the results obtained on the basis of expression stated in (13) are the best and values of $\Delta G$ derived on the basis of (13) are the closest to the experimental values even for large undercoolings. At $T=T_{\mathrm{g}}$ the error is only about $3.4 \%$ at the undercooling $\Delta T=370 \mathrm{~K}$, while it is $36.6 \%, 31.5 \%$ and $8.8 \%$, based on expressions proposed by Turnbull, Hoffman, and Thompson and Spaepen respectively. 


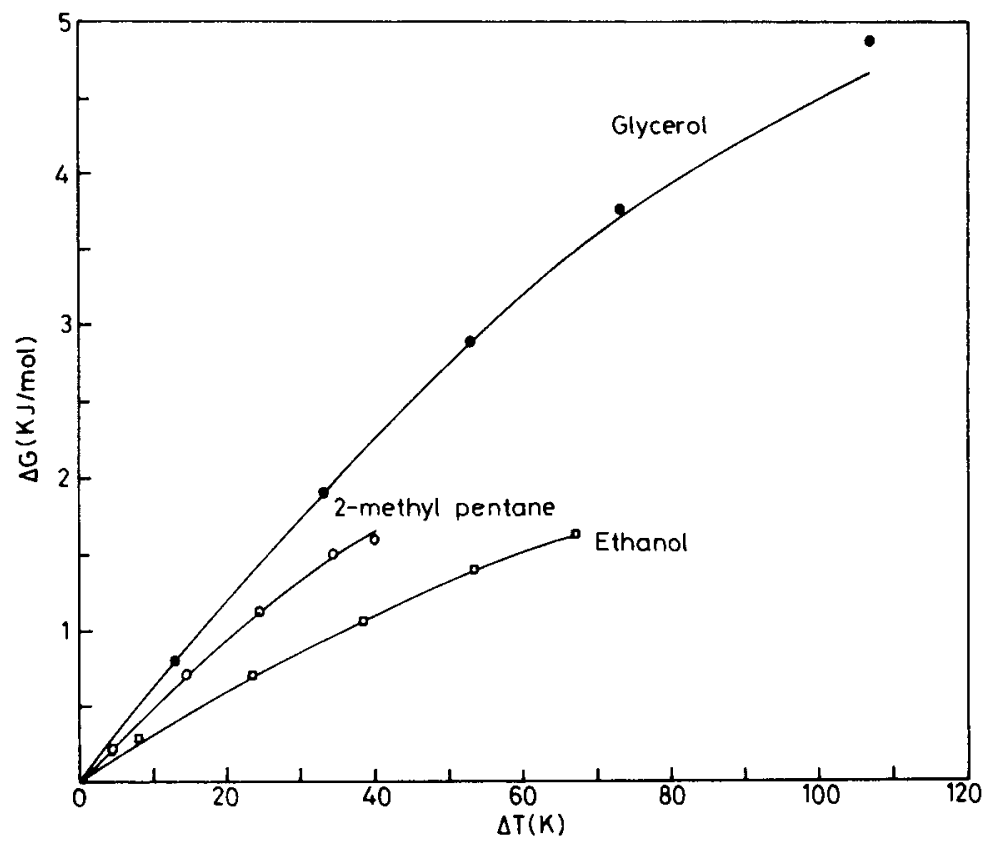

Figure 2. The Gibbs free energy difference, $\Delta G$ for molecular glass formers. Closed circles, open circles and squares represent experimental values of $\Delta G$ for glycerol, 2-methyl pentane and ethanol respectively whereas lines are calculated values of the respective samples.

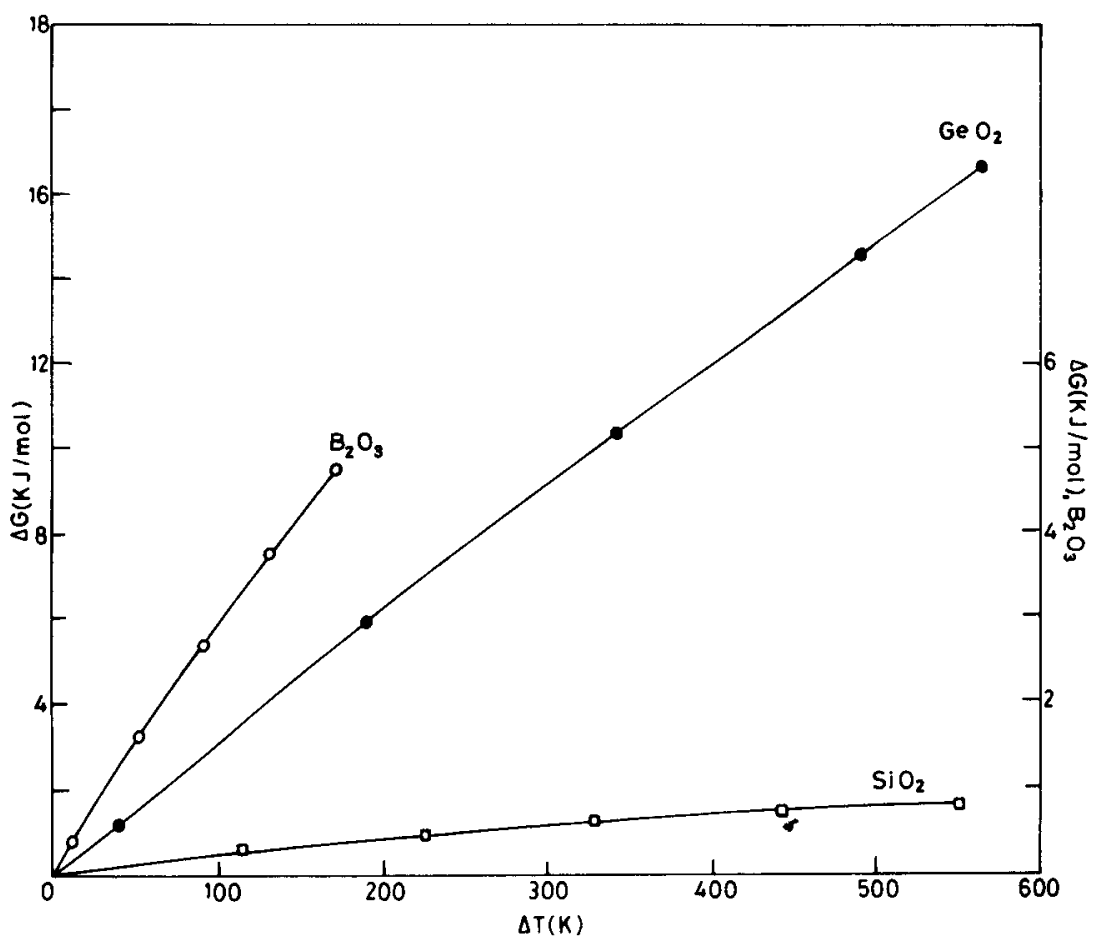

Figure 3. The Gibbs free energy difference, $\Delta G$, for oxide glass formers. Squares, closed circles and open circles represent experimental values of $\Delta G$ for $\mathrm{SiO}_{2}, \mathrm{GeO}_{2}$ and $\mathrm{B}_{2} \mathrm{O}_{3}$ respectively whereas lines are calculated values of the respective samples. 


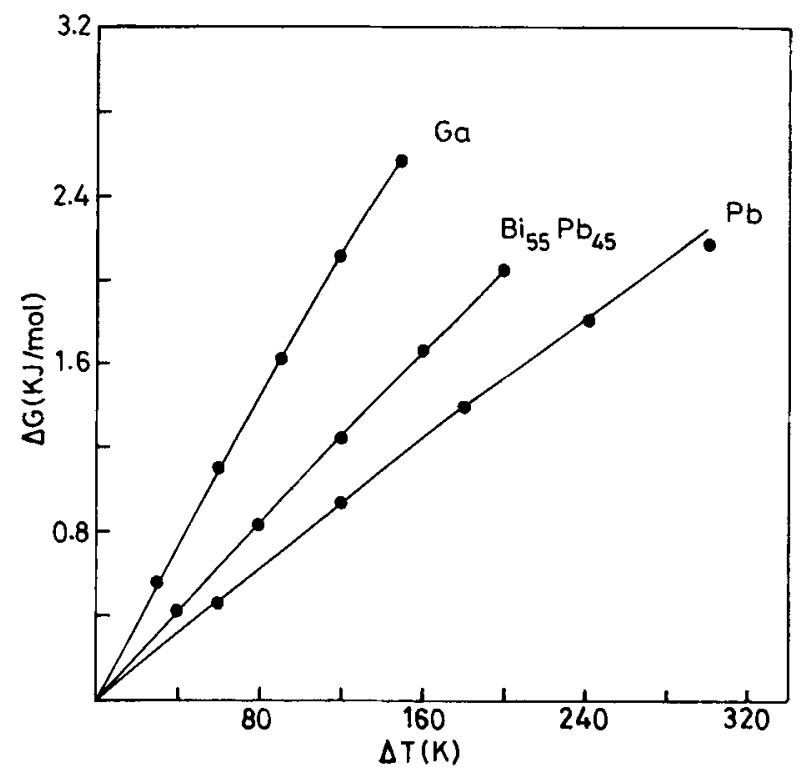

Figure 4. The Gibbs free energy difference, $\Delta G$, for non-glass forming melts. Closed circles and lines represent experimental and calculated values of $\Delta G$ of the respective samples.

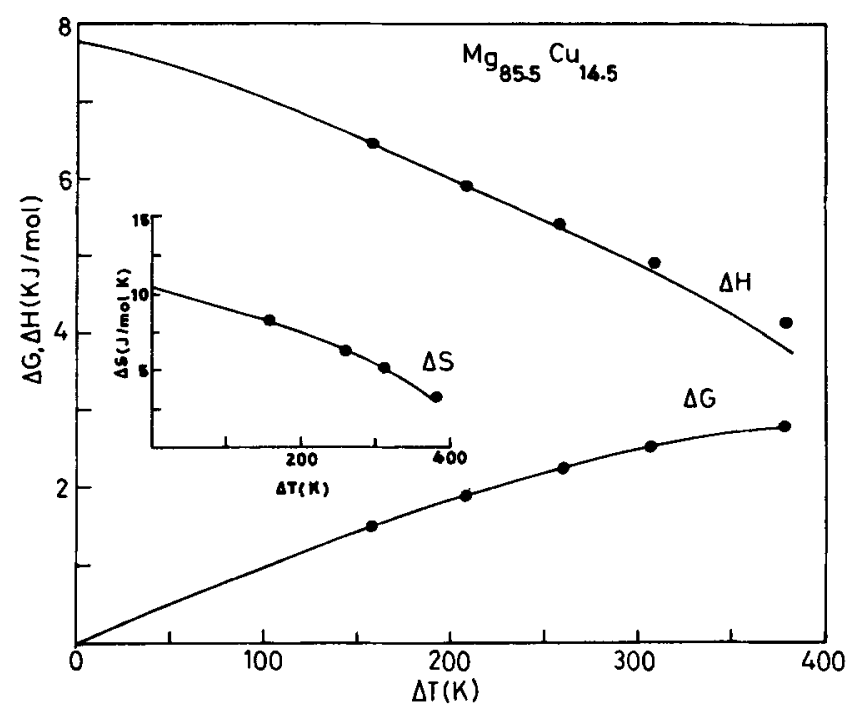

Figure 5. Variation of $\Delta G, \Delta H$ and $\Delta S$ with undercooling $\Delta T$ for metallic melt $\mathrm{Mg}_{85.5} \mathrm{Cu}_{14.5}$. Circles represent experimental data of respective parameters while lines are calculated values.

Thus, the expression for $\Delta G$ stated in (13) can be recommended in the use of nucleation and growth techniques. At the same time, it is interesting to note that present expression requires $\Delta C p^{m}, \Delta S_{\mathrm{m}}$ and $T_{\mathrm{m}}$ as input data which can be measured easily and more accurately and does not contain any adjustable parameter. 


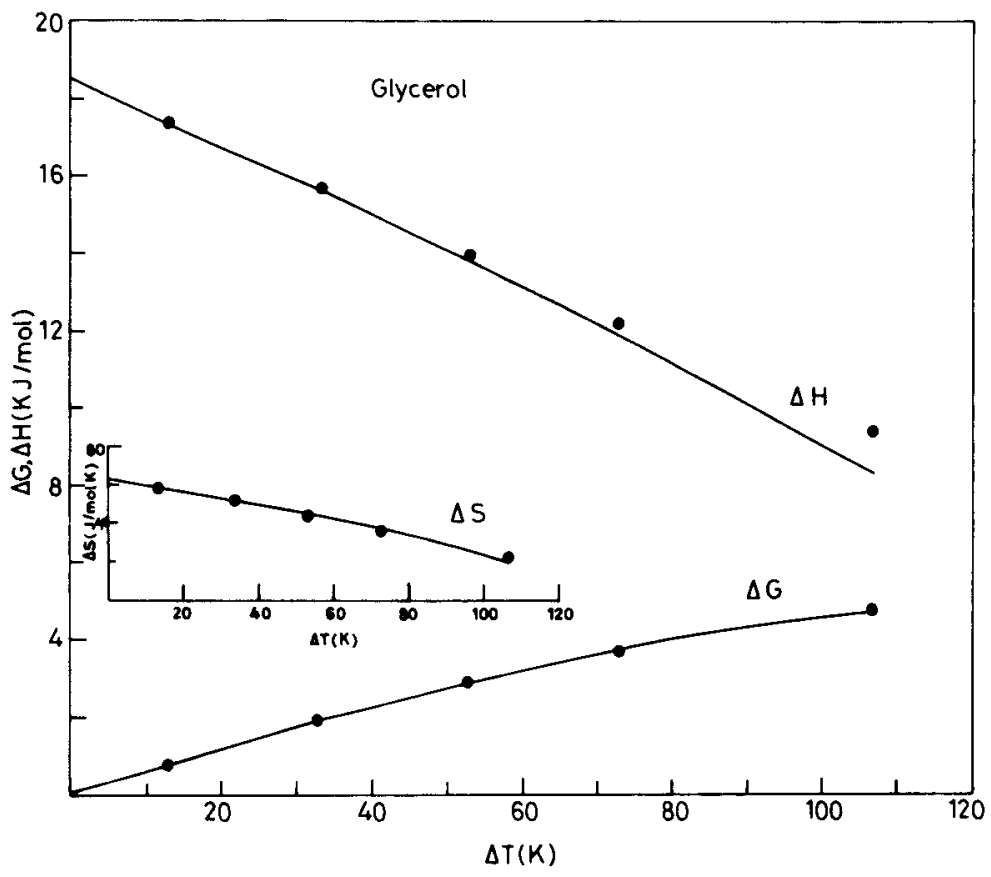

Figure 6. Variation of $\Delta G, \Delta H$ and $\Delta S$ with undercooling $\Delta T$ for molecular melt glycerol. Circles represent experimental values of respective parameters whereas lines are calculated values.

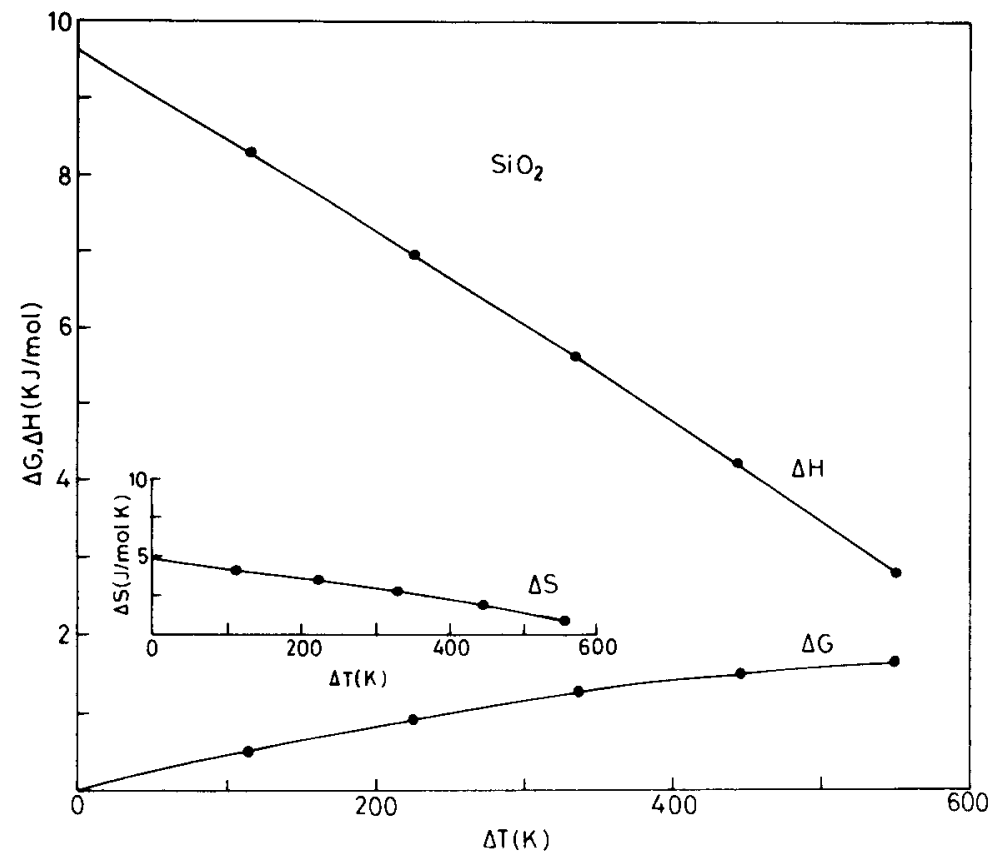

Figure 7. Variation of $\Delta G, \Delta H$ and $\Delta S$ with undercooling $\Delta T$ for oxide meit $\mathrm{SiO}_{2}$. Circles represent experimental values of respective parameters whereas lines are calculated values. 


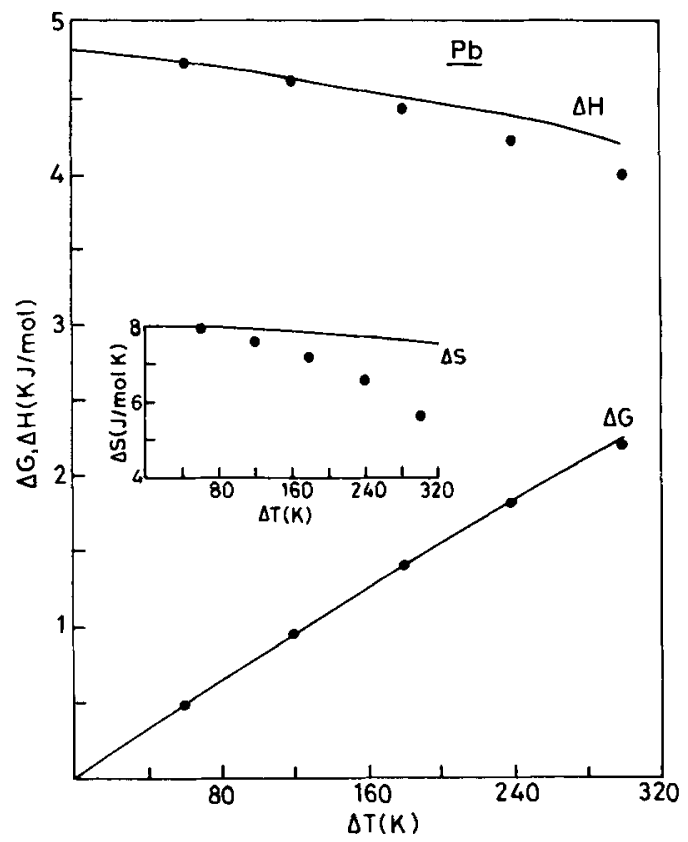

Figure 8. Variation of $\Delta G, \Delta H$ and $\Delta S$ with undercooling $\Delta T$ for non-glass forming melt $\mathrm{Pb}$. Circles represent experimental data of respective parameters while lines are calculated values.

The ideal glass transition temperature $T_{0}$ is calculated using (19) for glass forming melts and values obtained are reported in table 3 . From table 3 , it can be seen that the value of $T_{0}$ is very close to the extrapolated value of $T_{0}$ based on the experimental data. It can also be seen that the material with larger value of $\Delta C p^{m} / \Delta S_{\mathrm{m}}$ exhibits a larger value of $\delta=T_{0} / T_{\mathrm{m}}$. During the analysis, it is also found that $T_{0}$ is mainly controlled by the ratio $\Delta C p^{m} / \Delta S_{\mathrm{m}}$. An attempt has been made to relate $T_{\mathrm{g}}$ and $T_{0}$ and a linear relation

$$
T_{\mathrm{g}} / T_{\mathrm{m}}=a\left(T_{0} / T_{\mathrm{m}}\right)+b,
$$

is found between the reduced glass transition temperature and the reduced ideal glass transition temperature for each kind of glass forming melts. The values of constants $a$ and $b$ are reported in table 4 for metallic, molecular and oxide melts. A similar relation is also reported by Dubey and Ramachandrarao (1992) and Adam and Gibbs (1965) for organic materials.

The residual entropy $\left(\Delta S_{R}\right)$ of glass forming melts has been estimated and results are stated in table 3. $\Delta S_{\mathrm{R}}$ is also evaluated using experimental data of $\Delta C p$. It is found that calculated values of $\Delta S_{\mathrm{R}}$ is close to that based on experimental data. Attempt has also been made to seek a relation between $\Delta S_{\mathrm{R}}$ and characteristic temperatures $T_{0}$, $T_{\mathrm{g}}$ and $T_{\mathrm{m}}$. A linear relation has been obtained between $\Delta S_{\mathrm{R}} / \Delta S_{\mathrm{m}}$ and $\left(T_{\mathrm{g}}-T_{0}\right) / T_{\mathrm{m}}$ of the form

$$
\Delta S_{\mathrm{R}} / \Delta S_{\mathrm{m}}=m \frac{\left(T_{\mathrm{g}}-T_{0}\right)}{T_{\mathrm{m}}}+n
$$

where $m$ and $n$ are constants and are reported in table 4 . These constants are found to be different for different kinds of glass forming melts. From table 3, it can be realized 


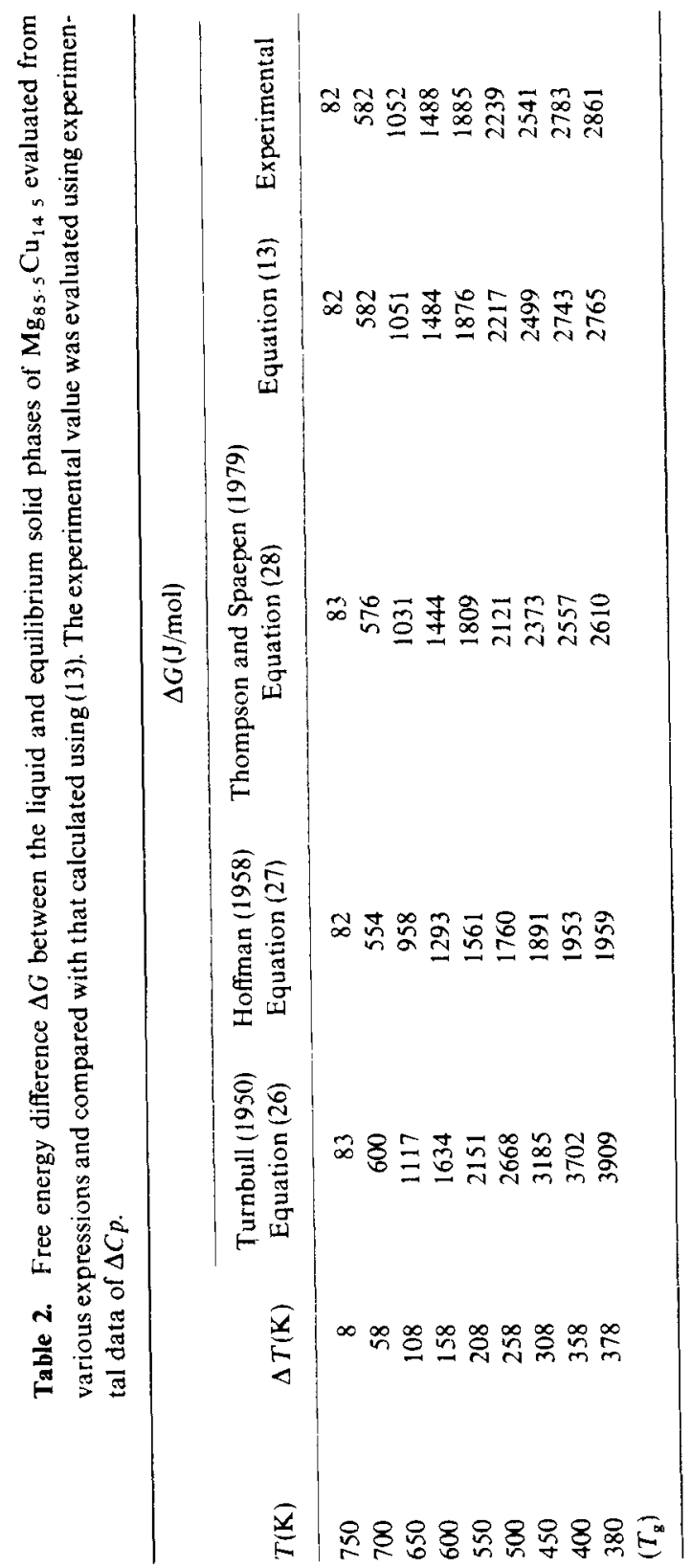

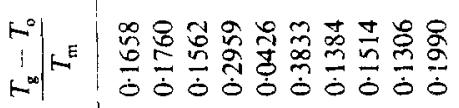

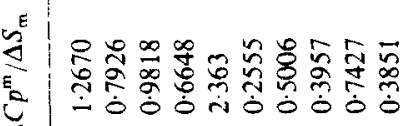

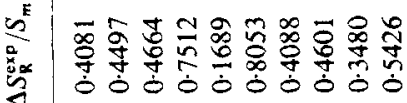

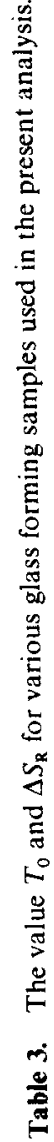

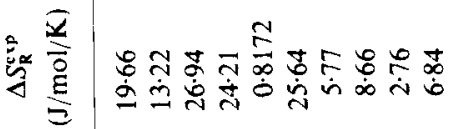

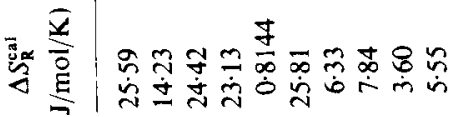

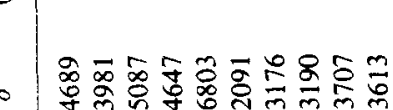

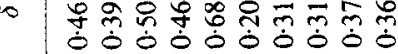

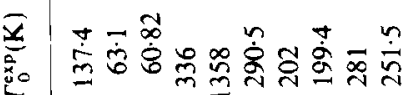

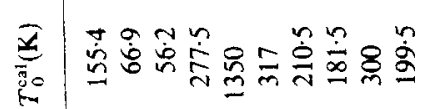

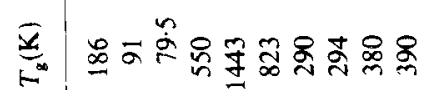

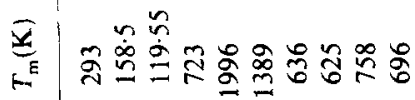

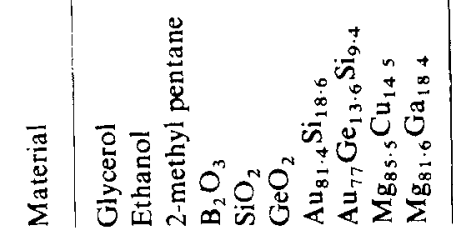


Table 4. The values of constants $a, b, m$, and $n$.

\begin{tabular}{lcccc}
\hline $\begin{array}{l}\text { Kinds of } \\
\text { materials }\end{array}$ & $a$ & $b$ & $m$ & $n$ \\
\hline Metallic & 1.27551 & 0.06056 & 2.5550 & 0.04423 \\
Molecular & 0.82521 & 0.24618 & -0.79223 & 0.57291 \\
Oxide & 0.28842 & 0.56185 & 1.96431 & 0.10252 \\
\hline
\end{tabular}

that material having smaller value of $\left(T_{\mathrm{g}}-T_{0}\right) / T_{\mathrm{m}}$ shows a lower value of $\Delta S_{\mathrm{R}} / \Delta S_{\mathrm{m}}$ which can be explained as follows. The material having lower value of $\Delta S_{\mathrm{R}}$ requires trapping of less amount of entropy to form a glass and such material can exhibit $T_{0}$ near $T_{\mathrm{g}}$ i.e. material shows less value of $\left(T_{\mathrm{g}}-T_{0}\right) / T_{\mathrm{m}}$. It can also be seen that the material with lower value of $\Delta S_{\mathrm{R}} / \Delta S_{\mathrm{m}}$ shows a larger value of $\Delta C p^{m} / \Delta S_{\mathrm{m}}$. According to earlier report of Dubey and Ramachandrarao (1984-85), the material having large value of $\Delta C p^{m} / \Delta S_{\mathrm{m}}$ is a good glass former. In view of these findings, one can say that the material with lower value of $\Delta S_{\mathrm{R}} / \Delta S_{\mathrm{m}}$ exhibits a large value of $\delta$ and $\Delta C p^{m} / \Delta S_{\mathrm{m}}$ and is a good glass former. Thus, it can be said that $\delta, \Delta C p^{m} / \Delta S_{\mathrm{m}}$ and $\Delta S_{\mathrm{R}} / \Delta S_{\mathrm{m}}$ are dependent on each other and can be used to measure the glass forming ability of materials.

\section{Conclusion}

The expression for $\Delta G$ reported in (13) is capable to estimate $\Delta G$ of all kinds of materials correctly at large undercooling also and expressions reported in (16) and (18) for $\Delta S$ and $\Delta H$ respectively can be used to study the temperature variation of these parameters. The value of the Kauzmann temperature $T_{0}$ is found to be close to the value extrapolated on the basis of experimental data. A linear relation is found between $T_{\mathrm{g}} / T_{\mathrm{m}}$ and $T_{0} / T_{\mathrm{m}}$ for glass forming melts. Both, reduced ideal glass transition temperature $\delta\left(=T_{0} / T_{\mathrm{m}}\right)$ and reduced residual entropy $\left(\Delta S_{\mathrm{R}} / \Delta S_{\mathrm{m}}\right)$ are mainly controlled by the parameter $\Delta C p^{m} / \Delta S_{\mathrm{m}}$ and $T_{\mathrm{m}}$. A linear relation is also found between $\Delta S_{\mathrm{R}} / \Delta S_{\mathrm{m}}$ and $\left(T_{\mathrm{g}}-T_{0}\right) / T_{\mathrm{m}}$. The material having lower value of $\Delta S_{\mathrm{R}} / \Delta S_{\mathrm{m}}$ can form glass more easily. $\Delta S_{\mathrm{R}}, T_{0}$ and $\Delta C p^{m} / \Delta S_{\mathrm{m}}$ are dependent on each other and the glass forming ability of materials can be studied in the form of these parameters.

\section{Acknowledgement}

The authors wish to express their thanks to Prof. P Ramachandrarao, NML, Jamshedpur and Prof. S Lele, Department of Metallurgical Engineering, BHU, Varanasi for their interests in the present work. Part of the work was done under the financial support by CSIR, New Delhi and authors are thankful for that.

\section{References}

Adam G and Gibbs J H 1965 J. Chem. Phys, 43139

Cohen M H and Grest G 1979 Phys. Rev. 3201077

Dubey K S and Ramachandrarao P 1984 Acta Metall. 3291

Dubey K S and Ramachandrarao P 1984-85 Int. J. Rapid Solidification 11 
Dubey K S and Ramachandrarao P 1992 Bull. Mater. Sci. 15111

Frenkel J 1955 Kinetic theory of liquids (New York: Dover)

Flory J 1953 Principles of polymer chemistry (New York: Cornell Univ. Press)

Gibbs J H and DiMarzio E A 1958 J. Chem. Phy's. 28373

Hirai N and Eyring H 1958 J. Appl. Phys. 29810

Hirai N and Eyring H 1959 Polymer Sci. 3751

Hoffman J D 1958 Chem. Phys. 291192

Jones D R H and Chadwick G A 1971 Philos. Mag. 24995

Kauzmann W 1948 Chem. Rer. 42219

Pandey S P, Dubey K S and Ramachandrarao P 1987 Key Engg. Mater. 13207

Sanchez I C 1974 J. Appl. Phys. 454204

Thompson C V and Spaepen F 1979 Acta Metall. 271855

Turnbull D 1950 J. Appl. Phys. 211022 